\title{
L'information géospatiale au service de l'agriculture africaine, un investissement essentiel pour les politiques agricoles
}

Jean-Philippe TONNEAU - Agnès BÉGUÉ - Louise LEROUX - Xavier AUGUSSEAU -

Jean-François FAURE - Benoit MERTENS - Camille PINET - Linda TOMASINI

Les informations géospatiales et leurs produits dérivés, conçus pour contribuer à la définition des politiques publiques agricoles, sont peu utilisés en Afrique. Les infrastructures, la formation et les compétences manquent ; les activités de recherche et développement sont dispersées et insuffisantes. Mais, surtout, les besoins restent mal formalisés et l'offre technologique, impulsée par les pays industrialisés, est peu adaptée aux caractéristiques des agricultures africaines. Par ailleurs, pour que des services opérationnels produisent de l'information pertinente et utile, un environnement stable doit être garanti aux entreprises, aux institutions et aux projets. Ces services co-construits naissent dans le cadre d'un partenariat pérenne entre les chercheurs, les opérateurs chargés des études et les utilisateurs finaux. La recherche ouvre alors d'autres voies d'exploitation des images satellitaires pour documenter et expliciter les processus de transformation de l'agriculture. Enfin, des réseaux de compétences interdisciplinaires structurés sur le temps long sont nécessaires pour favoriser les échanges méthodologiques et thématiques.

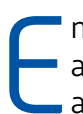

Afrique, les besoins en informations géospatiales actualisées pour accompagner les politiques publiques agricoles sont d'autant plus importants que les systèmes statistiques nationaux, basés généralement sur des inventaires, sont souvent défaillants. Le continent a cependant du mal à produire ces informations géospatiales. Les centres de formation aux technologies du spatial et les universités dispensant des cursus en télédétection (acquisition d'informations spatiales) et en géomatique (méthodes et outils d'analyse des données géographiques] sont peu nombreux et concentrés dans quelques pays.

Même là où ces centres existent, l'accès aux images satellitaires et aux outils de traitement n'entraîne pas un usage accru des données et informations géospatiales (voir encadré p. 2). Malgré les nombreux projets déployés sur le continent africain depuis la fin des années 2000, les services basés sur des données de télédétection restent rares, principalement par manque de compétences et d'interactions entre utilisateurs et producteurs d'informations.

De nombreux produits disponibles,

\section{au potentiel encore inexploité}

Les principaux domaines d'application en agriculture (voir figure p. 2) utilisent des produits dérivés de l'imagerie satellitaire qui sont calculés à partir de traitements pré-calibrés. Ces produits dits génériques regroupent
> les produits globaux de variables biophysiques de surface (surface foliaire, humidité du sol, température de surface, indices spectraux de végétation, etc.) et d'occupation des sols. Ils sont obtenus en routine sur l'ensemble du globe avec une résolution spatiale basse, de 250 mètres à plusieurs kilomètres. Un des exemples les plus connus est le produit annuel Land Cover de la Climate Change Initiative de l'Agence spatiale européenne (ESA) à 300 mètres de résolution spatiale, disponible depuis 1992

$>$ les cartes d'occupation des sols à plus fine résolution spatiale, produites à la demande sur de grandes étendues, souvent dans le cadre de projets. La Base de données d'occupation des terres (BDOT) du Burkina Faso au 1/100 000 est ainsi réalisée par l'Institut géographique burkinabé (IGB) avec l'assistance technique d'IGN FI, I'opérateur technique de l'Institut national de I'information géographique et forestière (ICN, France) pour ses projets à l'international ;

$>$ les spatiocartes, qui sont des images satellitaires en couleurs naturelles, géo-référencées, avec une surcouche incluant des éléments de paysage tels que routes, villes, cours d'eau, etc.

La plupart des produits génériques globaux sont issus de capteurs satellitaires à basse résolution. Ils sont principalement élaborés aux échelles régionale et nationale, et servent à la modélisation du cycle de l'eau, du cycle du carbone et du bilan d'énergie.

Les produits génériques d'occupation des sols permettent aussi de mesurer les changements comme la déforestation ou l'avancée de l'urbanisation. Ils ne sont cependant pas assez précis pour des 
besoins plus opérationnels à grande échelle $\left[1 / 5000^{\mathrm{e}}\right.$ à $\left.1 / 50000^{\mathrm{e}}\right]$, comme les plans d'affectation des sols, les statistiques agricoles ou l'agriculture de précision. Pour développer ces applications, il faut disposer de cartes d'occupation des sols à haute résolution spatiale fréquemment mises à jour. L'utilisation conjointe des données des satellites d'observation de la Terre Sentinel-2 de l'ESA et du satellite Landsat- 8 de la NASA devrait permettre d'élaborer une base de données d'occupation des sols équivalente à la base de données européenne CORINE Land Cover (CLC), produite dans le cadre du programme Copernicus, qui couvre 39 États [Europe élargie). La production de ces bases de données répond à des besoins prioritaires pour la majorité des pays africains.

La chaîne de l'information géospatiale va des données satellitaires à la prise de décision : pour fonctionner, elle doit non seulement s'appuyer sur les produits génériques existants mais aussi sur des produits thématiques et des services informationnels (voir figure p. 3).

Les produits thématiques, ou applications, sont conçus pour répondre à une question précise, territoriale ou environnementale - cartes de risque ou de potentiel, cartes d'indicateurs, tableaux de bord, etc. Ils intègrent des produits génériques et des connaissances thématiques, sous la forme de sources de données distinctes (statistiques, observations in situ) ou de traitements guidés par les experts en fonction de leurs savoirs et de leurs appréciations. Leur utilisation pour l'aide à la décision et la fourniture de services informationnels est contrainte par leur précision spatiale (résolution des images], temporelle [délai entre deux dates d'acquisition d'images), ou sémantique (classes thématiques de la légende).

Les services informationnels issus de ces produits thématiques s'adressent aux institutions chargées de l'élaboration et de la mise en œuvre des politiques agricoles et de gestion des ressources, aux

\section{L'imagerie satellitaire terrestre : abondante, en libre accès, mais nécessitant de fortes capacités de traitement}

L'observation de la Terre est un outil privilégié pour caractériser les surfaces agricoles aux échelles nationale et transcontinentale. Une centaine de satellites d'observation de la Terre sont en orbite et fournissent des données à différentes résolutions spatiales, de 30 centimètres à plusieurs kilomètres, à différentes fréquences de revisite (horaire, journalière, bimensuelle...) et dans des bandes spectrales variées et complémentaires.

L'accès aux données satellitaires à haute résolution spatiale (jusqu'à 10 mètres] est aujourd'hui facilité par des initiatives nationales et internationales, qui proposent des données gratuites [free access) ou en libre accès [open access], par exemple : le Pôle français de données et services pour les surfaces continentales (Theia, voir encadré p. 4], la Commission européenne [Copernicus, le programme d'observation de la Terre de l'Union européenne], le Cnes [Centre national d'études spatiales, France, via sa Plateforme d'exploitation des données Sentinel - PEPS), la National Aeronautics and Space Administration (NASA) aux États-Unis.

Le goulot d'étranglement, autrefois lié à l'acquisition des images et aux outils de traitement, s'est déplacé vers le volume de données à traiter. Un début de réponse est apporté par les technologies informatiques de type cloud-computing, qui permettent d'accéder à distance à des ressources de calcul et de traitement d'images, et par les logiciels libres de traitement d'images et de système $d^{\prime}$ 'information géographique $[S I G]$. Ces technologies requièrent toutefois de l'équipement, des connaissances et des compétences de plus en plus sophistiqués, ainsi qu'une connexion internet correcte. La démocratisation de l'accès aux données et aux outils est réelle, mais leur exploitation est liée à une technologie qui reste complexe et qui évolue vite. structures d'appui au secteur agricole et à certains producteurs agricoles. Ces utilisateurs finaux sont demandeurs d'informations récurrentes telles que les superficies emblavées, le suivi de la campagne agricole et les prévisions de rendements. Les services informationnels produisent de façon régulière et normalisée des informations facilement accessibles et utilisables. En conséquence, ces services obéissent à un cahier des charges précis, en fonction des besoins des utilisateurs finaux.

Les produits thématiques d'aide à la décision sont plus complexes à concevoir que les produits génériques parce qu'ils intègrent des données complémentaires et nécessitent d'adapter les méthodes aux conditions locales. Par exemple, les cartes de risque de maladies infectieuses vectorielles requièrent la connaissance de l'écologie des vecteurs pour mettre au point des indicateurs environnementaux pertinents. Les données complémentaires et l'expertise locale ne sont pas toujours disponibles, mais une assistance technique extérieure peut contribuer à développer cette expertise et à rendre possible l'appropriation locale des technologies. Aujourd'hui, d'autres produits thématiques utiles à plusieurs communautés sont générés en routine : c'est le cas des cartes d'anomalies de végétation, qui donnent des indications sur le niveau relatif de productivité de la végétation et qui sont intégrées à la plupart des systèmes d'alerte précoce pour la sécurité alimentaire.

\section{Améliorer la performance des produits existants, en impliquant la recherche dans des partenariats avec les utilisateurs}

Les raisons expliquant la faible performance des produits existants ou leur faible usage sont nombreuses - En premier lieu, les interactions avec les utilisateurs finaux font défaut. La stratégie d'offre de technologies, largement impulsée par les institutions du Nord, a pour objectif de transférer des données et des produits qui ne répondent que partiellement à la demande africaine. Les méthodes employées ne sont pas adaptées aux systèmes agricoles africains, plus diversifiés et beaucoup moins documentés que l'agriculture des pays industrialisés. Les conditions environnementales, la diversité des systèmes agricoles, le manque d'images et de données complémentaires, et les difficultés du partenariat sont autant de contraintes pour le transfert et l'adaptation.

Les principaux domaines d'application en agriculture utilisant les produits de la télédétection.

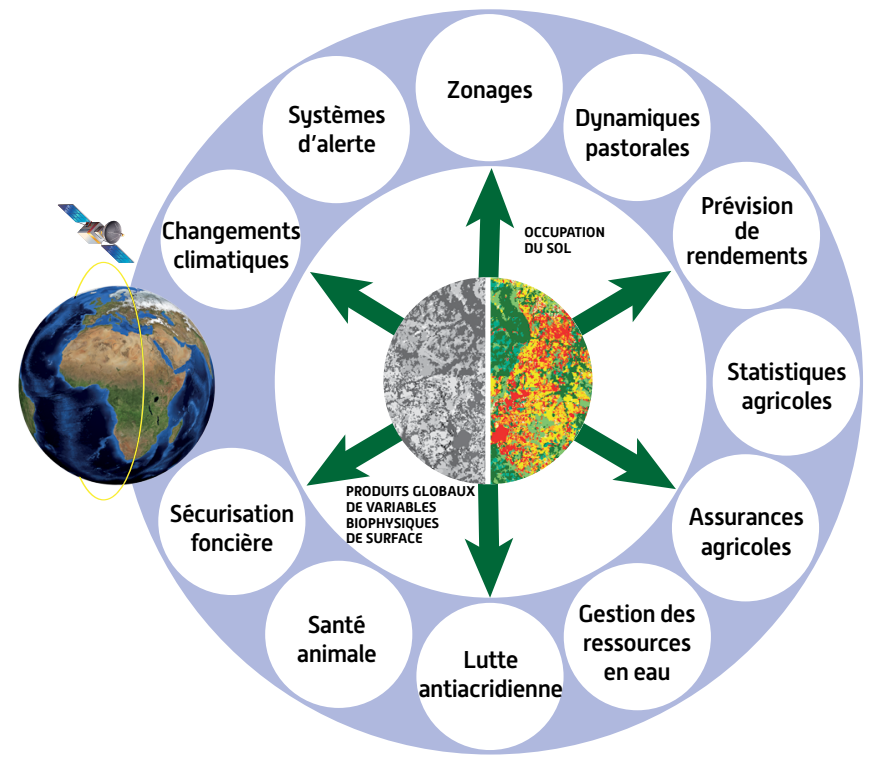


Co-construction de services informationnels géospatiaux : structurer une communauté de praticiens et d'utilisateurs.

C'est le besoin d'un service, via l'élaboration d'un cahier des charges et d'une analyse de marché, qui permet de définir les étapes nécessaires à sa création : quelles applications développer?
Quels produits génériques mobiliser pour développer ces applications? Quelles images utiliser pour obtenir ces produits?

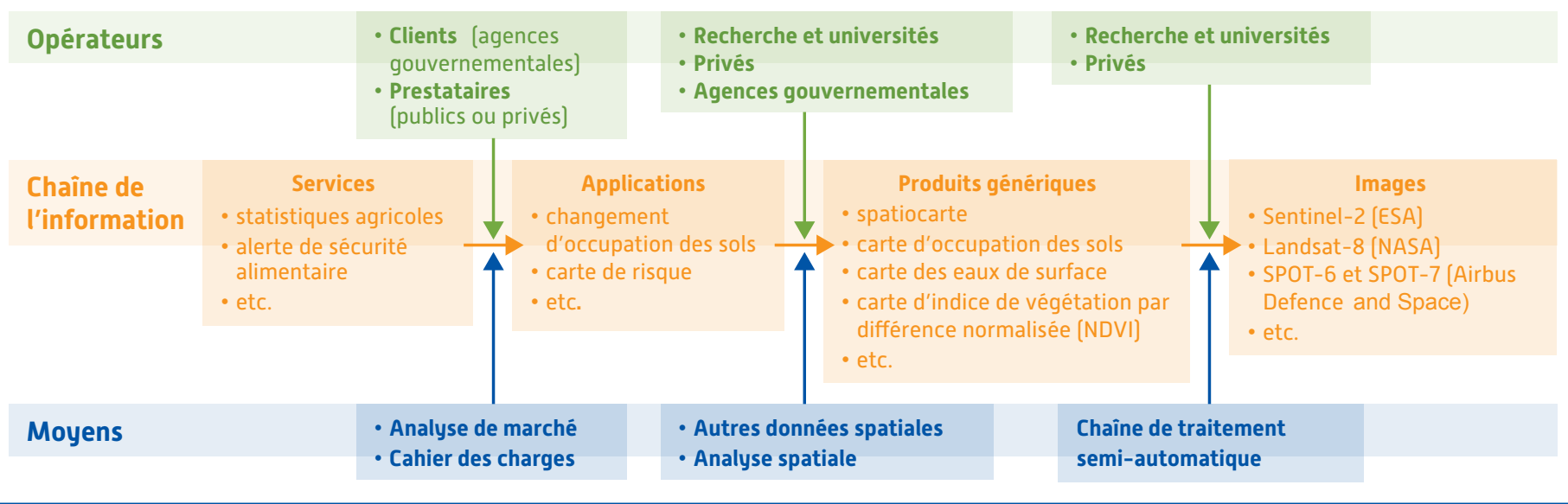

D'autres raisons existent, fragilisant la qualité de l'offre. La géomatique est une science jeune, nécessitant de la recherche appliquée. Or l'information géospatiale africaine souffre d'activités dispersées, principalement menées grâce à des projets ponctuels, souvent concurrents dans la recherche de financements. Elle s'est appuyée sur des projets de télédétection soutenus par des bailleurs de fonds internationaux, dans quelques pays, en direction d'institutions publiques nationales et régionales. Ces projets ont équipé des centres, formé des cadres et des techniciens, et lancé des applications pour le développement agricole. Mais la durée limitée des projets n'a pas toujours garanti la maintenance des équipements, la préservation des ressources humaines et la mise à niveau ou le transfert des compétences.

Le besoin de gouvernance et de coordination est évoqué par tous les acteurs pour les aspects d'équipements, d'infrastructures, de données et de formation. Le besoin de mutualisation, à travers une stratégie géomatique, nationale et régionale (grandes régions continentales) se fait sentir.

Mettre en place un environnement favorable pour garantir des services informationnels opérationnels [voir figure p. 3] - Pour se développer, la filière africaine d'information géospatiale a besoin que la télédétection et les technologies du spatial deviennent des outils reconnus à tous les niveaux de la prise de décision et de l'expertise pour le secteur agricole. Cela passe par des services informationnels opérationnels, fiables et aisés à mettre en œuvre en réponse aux besoins des acteurs du secteur.

Un service informationnel met en relation des besoins et des données dans un processus co-construit entre chercheurs, opérateurs chargés des études et utilisateurs finaux. Ce service s'appuie sur une chaîne d'activités articulant des fonctions de recherche, des fonctions de support technique, méthodologique et financier aux opérateurs intermédiaires, et des fonctions d'accompagnement des utilisateurs. Cette chaîne d'activités se déroule autant à l'étape de l'expression des besoins qu'à la mise en service proprement dite.

Pour que de tels services puissent être développés, un environnement général pérenne et stabilisé doit être assuré aux entreprises, aux institutions et aux projets. Les pouvoirs publics doivent garantir des investissements préalables allant dans ce sens. Des stratégies nationales de géomatique sont nécessaires pour encourager la mutualisation des données et des investissements humains et matériels, et ainsi éviter des investissements redondants. À l'image de la stratégie initiée au Sénégal (Plan national géomatique, PNG), elles peuvent assurer la coordination des actions, la définition d'un cadre réglementaire pour réguler la production et l'usage des données et des informations géographiques et, enfin, la pérennité technique et économique des services.

L'ensemble des images (images satellitaires diversifiées, récentes et historiques], les procédures de traitement adaptées et les capacités de calcul requises doivent être facilement accessibles. Les images et les produits génériques ou thématiques, souvent financés sur fonds publics, doivent être mutualisés afın de capitaliser l'existant et favoriser les innovations. La question de la gratuité de ces services se pose néanmoins : relèvent-ils du service public ou les utilisateurs doivent-ils participer financièrement? La mutualisation contribue de toute façon à diminuer les coûts.

Se doter d'une masse critique de techniciens, de professionnels et de chercheurs permettra aux pays africains de maîtriser et d'adapter les technologies, et de mettre au point des services en adéquation avec leur environnement socio-économique et géographique. Cela implique d'une part d'investir dans la formation initiale et professionnelle et, d'autre part, d'augmenter le nombre et la qualité des cursus. Ce renforcement des capacités bénéficiera également aux bureaux d'études et organisations non gouvernementales, aux côtés des instituts publics nationaux.

Faire en sorte que cet environnement soit au service des utilisateurs finaux - Un environnement favorable est une condition nécessaire mais non suffisante. II doit produire de l'information pertinente, utile dans les processus de prise de décision. Or les besoins des utilisateurs restent mal formalisés et l'offre existante est peu adaptée. Les produits thématiques et les services sont trop marqués par l'offre. La filière télédétection doit s'impliquer dans le co-développement de services géomatiques. Pour cela, des groupes de travail peuvent associer centres techniques, prestataires et utilisateurs, dans l'objectif de transformer les besoins en une demande formalisée et partagée, traduite dans un cahier des charges précis. En fonction de l'existant, des financements peuvent faciliter la mise à disposition de services déjà opérationnels (déploiement local, formation) ou la création d'applications ou de services répondant à des besoins exprimés n'ayant pas reçu de solution.

Structurer des réseaux de compétences - La recherche doit aller plus loin. Elle doit adapter les méthodes aux caractéristiques des agricultures africaines. Elle doit travailler en partenariat pour mettre au point de véritables services informationnels en adéquation avec les besoins : ce partenariat comprend notamment 
les bureaux d'études chargés de développer les produits d'aide à la décision et l'implication des acteurs locaux.

L'objectif est de structurer des réseaux de compétences favorisant les échanges méthodologiques et thématiques. Ces réseaux peuvent fonctionner sur le principe de communautés de pratiques. Ils réunissent des professionnels en géomatique et en d'autres disciplines nécessaires au développement des produits thématiques attendus. Les travaux doivent aboutir à des méthodes harmonisées et adaptées et à des référentiels-métiers - langages,

Ce Perspective $n^{\circ} 51$ est issu de travaux de recherche conduits par les auteur.es et leurs partenaires dans le cadre des entités suivantes:

$>$ la Maison de la télédétection à Montpellier, France (MTD,

https://www.teledetection.fr] ;

$>$ Theia, le pôle français de données et de services pour les surfaces continentales (https://www.theia-land.fr/), qui a pour objectif d'accroitre l'utilisation de la donnée spatiale par la communauté scientifique et les acteurs publics ;

$>$ GeoDEV, le réseau de pôles de compétences autour de l'observation spatiale des pays et territoires du Sud (Animation régionale du pôle Theia pour les pays du Sud, http://www.theia-land.art-geodev.fr] ;

$>$ le projet européen SIGMA (Stimulating Innovation for Global Monitoring of Agriculture, 2014-2017, http://www.geoglam-sigma.info], financé dans le cadre du septième programme-cadre européen FP7 ;

$>$ le projet OSFACO (Observation spatiale des forêts d'Afrique Centrale et de I'Ouest, 2016-2019, http://www.osfaco.org], financé par l'Agence française de développement (AFD, https://www.afd.fr]

Ce numéro de Perspective s'inscrit dans une réflexion globale des acteurs de la filière de l'information spatiale où la Maison de la télédétection joue un rôle moteur, en particulier pour l'accompagnement des utilisateurs finaux. méthodologies, pratiques et outils normalisés et communs. Les démarches interdisciplinaires ainsi mises en œuvre intègrent des méthodologies issues de disciplines variées (informatique, statistiques, traitement du signal, sciences humaines et sociales, sciences du vivant, sciences de la Terre), et des données d'origines diverses : images satellitaires disponibles en haute et basse résolutions spatiales et d'autres moyens d'observation - in situ, drones, nano-satellites.
Il s'appuie également sur les deux expertises suivantes

Bégué A., Charrier B., Torre C., Lo Seen D., Tonneau J.-P., Leroux L., Morant $P$. 2016. Observation spatiale pour l'agriculture en Afrique : potentiels et défis. Paris, AFD-CIRAD, collection Notes Techniques 12, 182 p. ISSN 2492-2838. https://www.afd.fr/fr/observation-spatiale-pour-lagriculture-en-afriquepotentiels-et-defis.

PARM, 2018. Sénégal - Étude de faisabilité pour l'investissement dans le système d'accès à l'information pour la gestion des risques agricoles. Rapport final - Janvier 2018. Une étude de faisabilité conduite par le Cirad pour PARM (Platform for Agricultural Risk Management) / IFAD (International Fund for Agricultural Development), Rome, Italie, 138 p.

http://p4arm.org/document/access-to-information-system-for-agriculturalrisk-management-in-senegal.

Parmi les publications issues de ces travaux, citons les suivantes :

Leroux L., Bégué A., Lo Seen D., D., Jolivot A., Kayitakire F., 2017. Driving forces of recent vegetation changes in the Sahel: Lessons learned from regional and local level analyses. Remote Sensing of Environment 191: 38-54.

https://doi.org/10.1016/j.rse.2017.01.014.

Leroux L., Baron C., Zoungrana B., Traoré S., Lo Seen D., Bégué A., 2016. Crop monitoring using vegetation and thermal indices for yield estimates: case study of a rainfed cereal in semi-arid West Africa. IEEE Journal of Selected Topics in Applied Earth Observations and Remote Sensing 9[1]: 347-362 https://doi.org/10.1109/JSTARS.2015.2501343.

\section{Quelques mots sur...}

Jean-Philippe Tonneau, Cirad, Umr TETIS (Territoire Environnement Télédétection Information spatiale, https://www.umr-tetis.fr/index.php/fr], Montpellier. Agronome et géographe, il étudie les conditions d'utilisation de l'information spatiale dans les projets d'aménagement et de développement territorial.

jean-philippe.tonneau@cirad.fr

Agnès Bégué, Cirad, Umr TETIS, Montpellier. Agronome, spécialisée en télédétection pour les applications agricoles dans les pays du Sud.

agnes.begue@cirad.fr

Louise Leroux, Cirad, Upr AIDA (Agroécologie et intensification durable des cultures annuelles, https://ur-aida.cirad.fr], Montpellier. Géographe, spécialisée en télédétection et modélisation pour les applications agricoles dans les pays du Sud.

louise.leroux@cirad.fr

Xavier Augusseau, Cirad, Umr TETIS, Montpellier. Géographe, spécialisé dans la conception de dispositifs d'observation mobilisant de l'information spatiale pour la planification et la gestion de projets de territoires dans les pays du Sud.

xavier.augusseau@cirad.fr

Jean-François Faure, Ird (Institut de recherche pour le développement, https://www.ird.fr], Umr ESPACE-DEV (Espace pour le développement, http://www.espace-dev.fr], Montpellier. Géographe, il est responsable de dispositifs de réception de données satellitaires et animateur de GeoDEV (http://www.theia-land.art-geodev.fr), le réseau de pôles de compétences autour de l'observation spatiale des pays et territoires du Sud, développé par le Pôle de données et de services pour les surfaces continentales Theia [https://www.theia-land.fr]

jean-francois.faure@ird.fr

Benoit Mertens, Ird, Umr ESPACE-DEV, Montpellier. Céographe, chef de projets visant le renforcement des capacités et l'accès aux données satellitaires en Afrique, en particulier sur le suivi des forêts.

benoit.mertens@ird.fr

Camille Pinet, IGN FI, Paris (http://www.ignfi.fr). Agronome spécialisée en traitement de l'information spatiale. Chef de projets pour le renforcement des capacités lié à l'utilisation des données satellitaires en Afrique, en particulier sur les questions environnementales et agricoles. cpinet@ignfi.fr

(c) 1 Cette œuvre est mise
à disposition selon les
termes de la licence

Pour citer ce document

Tonneau J.-P., Bégué A., Leroux L., Augusseau X Faure J.-F., Mertens B., Pinet C., Tomasini L., 2019. L'information géospatiale au service de l'agriculture fricaine, un investissement essentiel pour les politiques agricoles. Cirad, Montpellier, Perspective 51. https://doi.org/10.19182/agritrop/00067.
Linda Tomasini, Cnes [Centre national d'études spatiales, http://www.cnes.fr], Direction des applications, de l'innovation et de la science (DIA), Toulouse. Ingénieure responsable du développement des applications spatiales à l'international.

linda.tomasini@cnes.fr

\section{Quelques liens}

Agence spatiale européenne (ESA, European Space Agency). Sentinel-2 https://sentinel.esa.int/web/sentinel/missions/sentinel-2

Copernicus, programme d'observation de la Terre de l'Union européenne [European Union's Earth Observation Programme].

https://www.copernicus.eu/fr.

ESA Climate Change Initiative (CCI). Land Cover Project.

https://www.esa-landcover-cci.org.

GéoSénégal. Plan national géomatique (PNG)

http://www.geosenegal.gouv.sn/?-Le-PNG-.

Josserand H., Rembold F. (Eds), 2015. Conference Proceedings of the Information for Meeting Africa's Agricultural Transformation and Food Security Goals (IMAAFS) UN Conference, Addis Ababa (Ethiopia), 1-3 October 2014. https://ec.europa.eu/jrc/en/event/conference/information-meetingafrica\%E2\%80\%99s-agricultural-transformation-and-food-security-goalsimaafs.

Plateforme d'exploitation des produits Sentinel du Cnes (PEPS) https://peps.cnes.fr/rocket/\#/home.

\section{Directeur de la publication : Michel Eddi, Président directeur général du Cirad Rédacteur.e.s en chef : Patrick Caron, Umr ART-Dev $\begin{array}{ll}\text { LARECHERCHE AGRONOMIQUE } & \text { Rédacteur.e.s en chef: Patrick Caron, Umr } \\ \text { POUR LE DÉVELOPPEMENT } & \text { (Acteurs, ressources et territoires dans le }\end{array}$ 42, rue Scheffer 75116 Paris • France www.cirad.fr développement] \\ Cécile Fovet-Rabot, délégation à l'information scientifique et technique \\ Mise en pages et illustrations : Laurence Laffont \\ Diffusion : Christiane Jacquet, délégation à la communication}

www.cirad.fr/publications-ressources/edition/perspective-policy-brief perspactive ISSN-L 2275-9131 - Email : perspective@cirad.fr

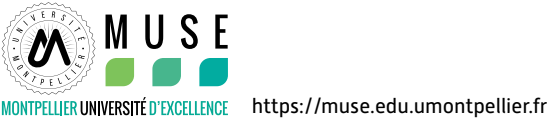

